\title{
Class I methanol masers in low-mass star formation regions
}

\author{
S. V. Kalenskii ${ }^{1}$, V. I. Slysh ${ }^{1}$, L. E. B. Johansson ${ }^{2}$, \\ P. Bergman ${ }^{2}$, S. Kurtz ${ }^{3}$, P. Hofner ${ }^{4}$, and C. M. Walmsley ${ }^{5}$ \\ ${ }^{1}$ Astro Space Center, Lebedev Physical Institute, 84/32 Profsoyuznaya st., Moscow, 117997, \\ Russia \\ email: kalensky@asc.rssi.ru \\ ${ }^{2}$ Onsala Space Observatory, Chalmers University of Technology, 43992 Onsala, Sweden \\ email: pbergman@chalmers.se \\ ${ }^{3}$ Centro de Radioastronomía y Astrofísica, Universidad Nacional Autonoma de México \\ (Morelia, Michoacán, México) \\ email: s.kurtz@crya.unam.mx \\ ${ }^{4}$ Physics Department, New Mexico Tech., 801 Leroy Pl., Socorro, NM 87801, and National \\ Radio Astronomy Observatory, Socorro, NM 87801, USA \\ email: hofner_p@yahoo.com \\ ${ }^{5}$ Osservatorio Astrofisico di Arcetri, Largo E. Fermi 5,1-50125 Firenze, Italy \\ email: walmsley@arcetri.astro.it
}

\begin{abstract}
Four Class I maser sources were detected at 44, 84, and $95 \mathrm{GHz}$ toward chemically rich outflows in the regions of low-mass star formation NGC 1333I4A, NGC 1333I2A, HH25, and L1157. One more maser was found at $36 \mathrm{GHz}$ toward a similar outflow, NGC 2023. Flux densities of the newly detected masers are no more than $18 \mathrm{Jy}$, being much lower than those of strong masers in regions of high-mass star formation. The brightness temperatures of the strongest peaks in NGC 1333I4A, HH25, and L1157 at $44 \mathrm{GHz}$ are higher than $2000 \mathrm{~K}$, whereas that of the peak in NGC 1333I2A is only 176 K. However, a rotational diagram analysis showed that the latter source is also a maser. The main properties of the newly detected masers are similar to those of Class I methanol masers in regions of massive star formation. The former masers are likely to be an extension of the latter maser population toward low luminosities of both the masers and the corresponding YSOs.
\end{abstract}

Keywords. masers, ISM: jets and outflows, ISM: molecules

\section{Introduction}

In spite of a number of observations and theoretical works, the nature of Class I methanol masers is still unknown. This is partly because until recently these masers have been observed only in regions of massive star formation, which are typically distant (2-3 kpc from the Sun or farther) and highly obscured at optical and even NIR wavelengths. In addition, high mass stars usually form in clusters. These properties make it difficult to resolve maser spots and to associate masers with other objects in these regions. In contrast, regions of low-mass star formation are much more widespread and many of them are only 200-300 pc from the Sun; they are less heavily obscured than regions of high-mass star formation, and there are many isolated low-mass protostars. Therefore, the study of masers in these regions might be more straightforward compared to that of high-mass regions, and hence, the detection of Class I masers there might have a strong impact on maser exploration. 

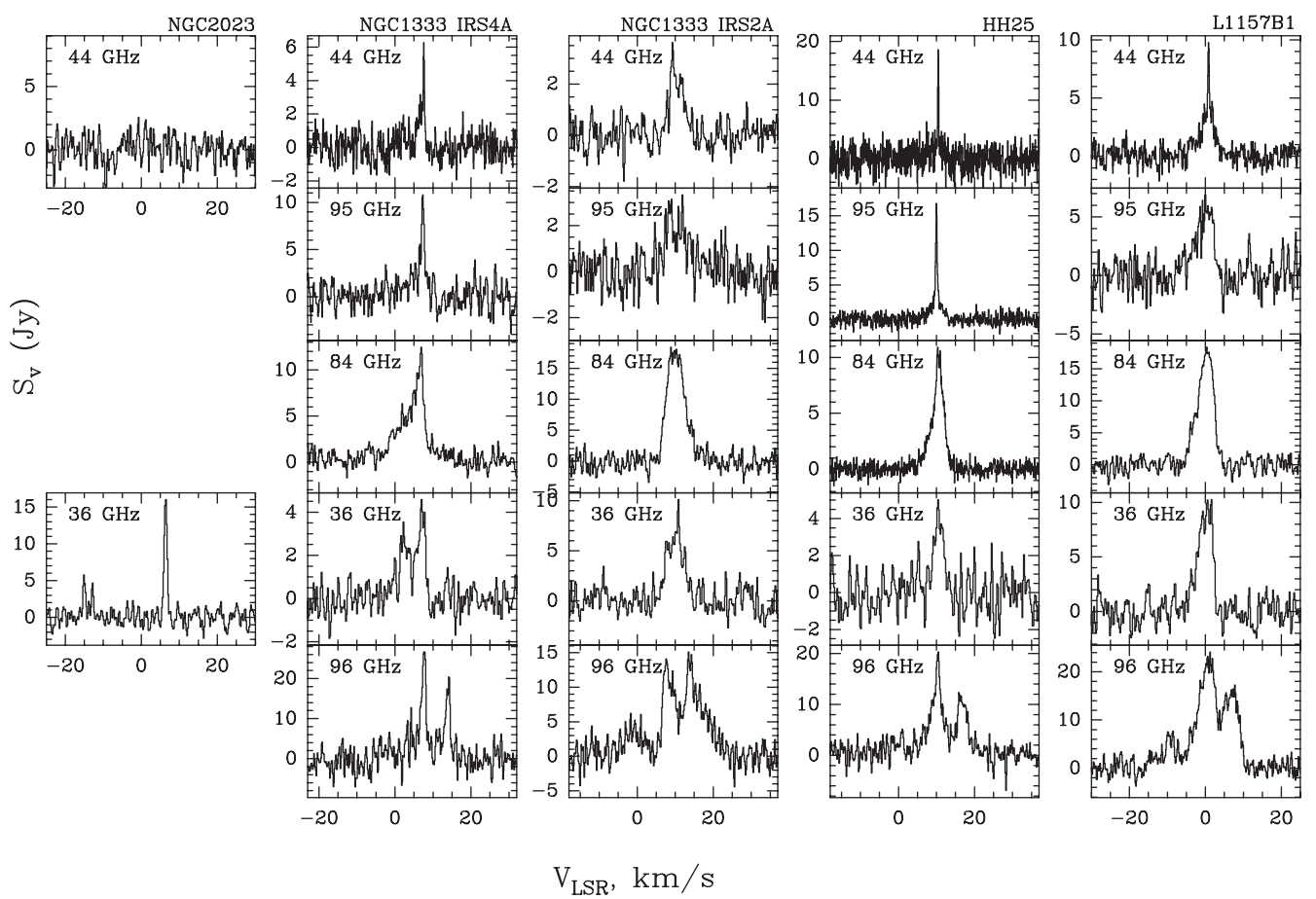

Figure 1. Spectra of the newly detected masers.

Bearing this in mind, we undertook a search for Class I methanol masers in regions of low-mass star formation. Since the most common viewpoint is that these masers arise in postshock gas in the wings of bipolar outflows (Plambeck \& Menten, 1990; Chen et al. 2009) our source list was composed of these objects. The naive expectation is to find methanol masers towards bright thermal sources of methanol; therefore the basis of our source list consists of "chemically rich outflows", where methanol abundance is significantly enhanced relative to that in quiescent gas. Because methanol enhancement has been detected in young, well-collimated outflows from Class 0 and I sources, we included several such objects in our list regardless of whether methanol enhancement had been previously found there. A subsample of our list consisted of YSOs with known outflows and/or $\mathrm{H}_{2} \mathrm{O}$ masers located in Bok globules. Like other objects from our list, these YSOs are typically isolated objects of low or intermediate mass, located in nearby $(<500 \mathrm{pc})$ small and relatively simple molecular clouds. In total, our source list consisted of 37 regions which harbor 46 known outflows driven by Class 0 and I low-mass protostars, taken from the literature. All of them were observed in the $7_{0}-6_{1} A^{+}$transition at $44 \mathrm{GHz}$, where the strongest Class I masers have been found so far. In addition to the $7_{0}-6_{1} A^{+}$transition, most sources were observed in other Class I maser lines, namely, in the $4_{-1}-3_{0} E$ line at $36 \mathrm{GHz}$, in the $5_{-1}-4_{0} E$ line at $84 \mathrm{GHz}$, and in the $8_{0}-7_{1} A^{+}$ line at $95 \mathrm{GHz}$, as well as the "purely thermal" $2_{K}-1_{K}$ lines at $96 \mathrm{GHz}$.

\section{Observations and results}

Single-dish observations. The single-dish observations are described in detail by Kalenskii et al. (2006, 2010a). They were carried out with the 20-m radio telescope of the Onsala Space Observatory (OSO) during several observing sessions in 2004-2011. As a result, we detected maser candidates at $44 \mathrm{GHz}$ towards NGC 1333I2A, NGC 1333I4A, HH 25 
Table 1. Parameters of maser sources determined by the VLA observations.

\begin{tabular}{|c|c|c|c|c|c|c|}
\hline Source & $\begin{array}{c}\text { R.A. } \\
(\text { J2000) }\end{array}$ & $\begin{array}{c}\text { Dec. } \\
(\mathrm{J} 2000)\end{array}$ & $\mid \begin{array}{c}\text { Major } \\
\text { axis (") }\end{array}$ & $\mid \begin{array}{c}\text { Minor } \\
\left.\text { axis (" }{ }^{\prime \prime}\right)\end{array}$ & $\begin{array}{c}T_{\text {B R }} \\
(\mathrm{K})\end{array}$ & $\begin{array}{c}\mathrm{V}_{\text {lsr }} \\
\left(\mathrm{km} \mathrm{s}^{-1}\right)\end{array}$ \\
\hline $\mathrm{NGC} 1$ & $\mid \begin{array}{ll}03290\end{array}$ & 31142 & 2 & 1 & 170 & 10.9 \\
\hline NGC $1333 \mathrm{I} 2 \mathrm{~A}$ M2 & $\begin{array}{lll}03 & 29 & 01.422\end{array}$ & 31141 & 2 . & 2. & 35 & 8.8 \\
\hline NGC 1333I4A & $\begin{array}{lll}03 & 29 & 10.829\end{array}$ & $\begin{array}{lll}31 & 13 & 18.68\end{array}$ & 1. & 0.4 & 2400 & 6.9 \\
\hline $5 \mathrm{M} 1$ & $\begin{array}{lll}05 & 46 & 08.071\end{array}$ & -001405.66 & 2 & 0 & inf & 9.6 \\
\hline $\mathrm{HH} 25 \mathrm{M} 2$ & $\begin{array}{lll}05 & 46 & 07.967\end{array}$ & $-00 \quad 14 \quad 02.42$ & 0 & 0 . & $\inf$ & 9.6 \\
\hline L1157 M1 & $\begin{array}{llll}20 & 39 & 10.033\end{array}$ & $\begin{array}{lll}68 & 01 & 42.20\end{array}$ & 0 . & 0 . & 53000 & 0.8 \\
\hline L1157 M2 & 203909.465 & $\begin{array}{lll}68 & 01 & 15.59\end{array}$ & 1.9 & 0.7 & 470 & 1.7 \\
\hline
\end{tabular}

and L1157. Toward NGC 1333I4A and HH 25, narrow features were also found at 95 and $84 \mathrm{GHz}$. In addition, a narrow line was detected at $36 \mathrm{GHz}$ toward the blue lobe of an extremely high-velocity outflow in the vicinity of the bright reflection nebula NGC 2023. The source spectra are shown in Fig. 1.

VLA/EVLA observations. To check whether the newly detected sources are really masers we observed them with the NRAO $\dagger$ VLA/EVLA array in the D configuration, which provides an angular resolution about $1 .^{\prime \prime} 5$ at $44 \mathrm{GHz}$. L1157 was observed with the VLA on March 17, 2007; the other sources were observed with the EVLA on August 08, 2010. The data were reduced using the NRAO Astronomical Image Processing System (AIPS) package. The source parameters are presented in Table 1.

\section{Are the newly detected sources really masers?}

The small sizes and high brightness temperatures at $44 \mathrm{GHz}$ indicate that the newly detected sources are masers. The exceptions are NGC 2023, which was not found at $44 \mathrm{GHz}$, and NGC 1333I2A, with a line brightness temperature of only $170 \mathrm{~K}$ (Table 1). The nature of the $36 \mathrm{GHz}$ line in the blue lobe of the bipolar outflow in NGC 2023 is unclear. On the one hand, the line is fairly narrow, and offset measurements showed that the source is compact at least with respect to the 105-arcsec Onsala beam. These properties suggest that the source is a maser. This assumption has further support in the fact that the line LSR velocity, $\approx 6.5 \mathrm{~km} \mathrm{~s}^{-1}$, is less than the systemic velocity of about $10 \mathrm{~km} \mathrm{~s}^{-1}$. On the other hand, the line has no counterpart at $44 \mathrm{GHz}$, which is more typical for thermal emission. Note, however, that there are known masers at $36 \mathrm{GHz}$ without 44-GHz counterparts; in particular, no $44 \mathrm{GHz}$ emission was found at the velocity of a fairly strong $36-\mathrm{GHz}$ maser detected $3^{\prime}$ north of DR21(OH) by Pratap et al. (2008). Therefore, we tentatively conclude that the narrow line in NGC 2023 is a maser.

The fairly low brightness temperature and finite sizes (Table 1) of NGC 1333I2A (M1 and M2) suggest that they are thermal sources. However, a rotational diagram analysis (Kalenskii et al., in prep.) shows that they are low-gain masers or a cluster of weak masers.

\section{Properties of the new masers}

Association with chemically rich outflows. New masers were found towards the lobes of outflows in NGC 1333I4A, NGC 1333I2A, NGC 2023, HH25, and L1157. These outflows are known to be chemically rich outflows with enhanced methanol abundances.

$\dagger$ The National Radio Astronomy Observatory is operated by Associated Universities, Inc., under contract with the National Science Foundation. 
Comparison of the VLA maps with high-resolution maps of thermal methanol and other molecules shows that the masers coincide with chemically rich gas clumps, where the abundances of methanol and other molecules are enhanced (e.g., Gibb \& Davis (1988); Bachiller et al. (1998); Benedettini et al. (2007)). In L1157, the masers are located in gas clumps, which, according to chemical modeling of Viti et al. (2004), probably pre-existed the outflow.

LSR velocities and intensities. Comparison of the maser LSR velocities with those of thermal methanol lines, observed in the same directions, show that these velocities coincide within $0.5 \mathrm{~km} / \mathrm{s}$. This coincidence occurs even when the LSR velocities of some other molecular lines toward the maser positions are significantly different. The LSR velocities of both maser and thermal methanol lines are usually close to the systemic velocities. An exception is the $36-\mathrm{GHz}$ maser in the EHV outflow NGC 2023. Its radial velocity is less than the systemic velocity by about $3.5 \mathrm{~km}^{-1}$. Note that Voronkov (this volume) has detected a high-velocity Class I maser just at $36 \mathrm{GHz}$.

Maser intensities. The new masers are weaker than the bright masers typical in regions of massive star formation. However, they obey the same relationship between the maser and YSO luminosities as reported by Bae et al. (2011) for masers in regions of high- and intermediate-mass star formation, thus extending this relationship toward low luminosities (Kalenskii et al., in prep).

Variability. Several sessions of repeated observations of NGC 1333I4A, HH25, and L1157 at $44 \mathrm{GHz}$ were performed in 2008-2011. No notable variations were found. Slight changes in line intensities can be attributed to poor signal-to-noise ratios and calibration uncertainties. However, further monitoring of these sources is desirable in order to search for flares similar to that which occurred in DR $21(\mathrm{OH})$.

To summarize, the main properties of the newly detected masers are similar to those of Class I methanol masers in regions of massive star formation. The former masers are likely to be an extension of the latter maser population toward lower luminosities of both the masers and the corresponding YSOs.

\section{Maser models}

The fact that the maser LSR velocities coincide with the systemic velocities allows us to conclude that the masers appear in dense clumps of gas, probably pre-existing the outflows. However, the exact nature of the masers remains unknown. Sobolev et al. (1998) suggested that compact maser spots arise in extended, turbulent clumps because in a turbulent velocity field the coherence lengths along some directions are larger than the mean coherence length, resulting in a random increase of the optical depth absolute values along certain sight lines in a clump. According to Kalenskii et al. (2010b) such a model can easily explain the observed brightness of the maser lines, but within the framework of this model it is difficult to explain why single peaks dominate the maser emission in the L1157 clumps. However, natural additional assumptions, such as the existence of shocks or centrally condensed clumps, makes it possible to explain the observational data.

An examination of the maser spectra in L1157 may lead to another interpretation of our results. Both $44 \mathrm{GHz}$ masers detected in this source have double line profiles. It is known that a double thermal line with a "blue asymmetry" may be a signature of collapse (Zhou (1996)). Contrary to this, the masers in L1157 exhibit a "red asymmetry". However, just such an asymmetry is what one would expect for Class I masers arising in a collapsing clump. This model is discussed in more detail by Kalenskii et al. (2010b). Note that this model, if correct, is specific for the masers in L1157; no other maser in our sample exhibits a double line profile. 


\section{Acknowledgements}

The work was financially supported by RFBR (grants No. 04-02-17547, 07-02-00248, and 10-02-00147-a), and Federal National Scientific and Educational Program (project number 16.740.11.0155). P.H. acknowledges partial support from NSF grant AST 0908901. S.Kurtz acknowledges support from UNAM DGAPA grant IN101310. The Onsala Space Observatory is the Swedish National Facility for Radio Astronomy and is operated by Chalmers University of Technology, Göteborg, Sweden, with financial support from the Swedish Research Council and the Swedish Board for Technical Development.

\section{References}

Bachiller, R., Codella, C., Colomer, F., Liechti, S., \& Walmsley, C. M. 1998, A\&6A, 335, 266

Bae, J.-H., Kim, K.-T., Youn, S.-Y., Kim, W.-J., Byun, D.-Y., Kang, H., \& Oh, C. S., 2011, ApJS 196, 21

Benedettini, M., Viti, S., Codella, C., Bachiller, R., Gueth, F., Beltran, M. T., Dutrey, A., \& Guilloteau, S. 2007, Mon. Not. R. Astron. Soc., 381, 1127

Chen, X., Ellingsen, S. P., \& Shen, Z.-Q. 2009, Mon. Not. R. Astron. Soc., 396, 1603

Gibb, A. \& Davis, C. J. 1998, Mon. Not. R. Astron. Soc., 298, 644

Kalenskii, S. V., Promyslov, V. G., Slysh, V. I., Bergman, P., \& Winnberg, A. 2006, Astron. Rep., 50, 289

Kalenskii, S. V., Johansson, L. E. B., Bergman, P., Kurtz, S., Hofner, P., Walmsley, C. M., \& Slysh, V. I., 2010, Mon. Not. R. Astron. Soc., 405, 613

Kalenskii, S. V., Kurtz, S., Slysh, V. I., Hofner, P., Walmsley, C. M., Johansson, L. E. B., \& Bergman, P. 2010, Astron. Rep., 54, 932

Plambeck, R. L. \& Menten, K. M. 1990, ApJ, 364, 555

Pratap, P., Shute, P. A., Keane, T. C., Battersby, C., \& Sterling S. 2008, AJ, 135, 1718

Sobolev, A. M., Wallin, B. K., \& Watson, W. D. 1998, ApJ, 498, 763

Zhou S. 1996, in van Dishoeck E. F., ed, Proc. IAU Symp. 178, Molecules in Astrophysics: Probes and Processes, Kluwer, Dordrecht, p. 195

Viti, S., Codella, C., Benedettini, M., \& Bachiller, R. 2004, Mon. Not. R. Astron. Soc., 350, 1029 